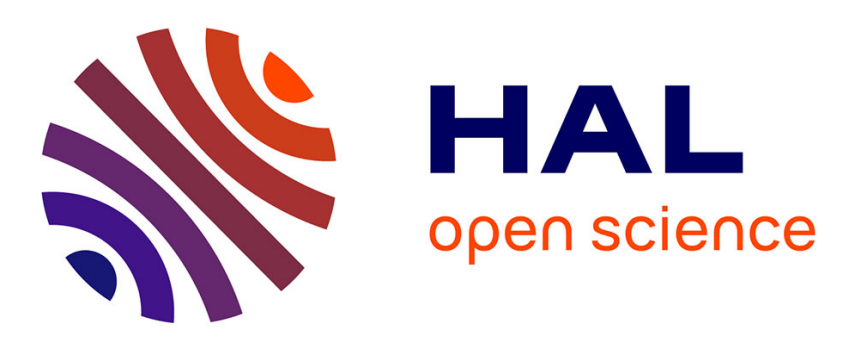

\title{
Stabilisation des systèmes globalement asymptotiquement commandables
}

\author{
Ludovic Rifford
}

\section{To cite this version:}

Ludovic Rifford. Stabilisation des systèmes globalement asymptotiquement commandables. Comptes rendus de l'Académie des sciences. Série I, Mathématique, 2000, 330 (3), pp.211-216. hal-00768990

\section{HAL Id: hal-00768990 \\ https://hal.science/hal-00768990}

Submitted on 27 Dec 2012

HAL is a multi-disciplinary open access archive for the deposit and dissemination of scientific research documents, whether they are published or not. The documents may come from teaching and research institutions in France or abroad, or from public or private research centers.
L'archive ouverte pluridisciplinaire HAL, est destinée au dépôt et à la diffusion de documents scientifiques de niveau recherche, publiés ou non, émanant des établissements d'enseignement et de recherche français ou étrangers, des laboratoires publics ou privés. 
Contrôle optimal / Optimal Control

\title{
Stabilisation des systèmes globalement asymptotiquement com- mandables
}

\section{Ludovic Rifford}

Institut Girard Desargues, Université Claude Bernard Lyon I

43, boulevard du 11 Novembre 1918, 69622 Villeurbanne Cedex.

Courriel: rifford@desargues.univ-lyon1.fr

Résumé. Etant donné un système commandé globalement asymptotiquement commandable à l'origine, nous déduisons d'un théorème d'existence de fonction Lyapunov semiconcave, la construction d'un retour d'état globalement asymptotiquement stabilisant au sens des solutions Euler. Celuici se trouve être assez régulier dans le cas des systèmes affines en la commande.

\section{Stabilization of globally asymptotically controllable systems}

\begin{abstract}
Given a globally asymptotically controllable system, we derive from an existence theorem of a semiconcave control-Lyapunov function, the construction of a globally asymptotically stable feedback in the Euler sense. The feedback is rather regular in the case of control systems affine in the control.
\end{abstract}

\section{Abridged English Version}

In this paper, we study systems of the general form

$$
\dot{x}(t)=f(x(t), u(t))
$$

where the state $x(t)$ takes values in a Euclidian space $\mathbb{R}^{n}$, the control $u(t)$ takes values in a given compact set $U$, and $f$ is locally Lipschitz in $(x, u)$. A special element " 0 " is distinguished in $U$, and the state $x=0$ of $\mathbb{R}^{n}$ is an equilibrium point, i.e., $f(0,0)=0$. A trajectory will be an absolutely continuous function $x(\cdot):[a, b] \rightarrow \mathbb{R}^{n}$ associated to a control $u(\cdot):[a, b] \rightarrow \mathbb{R}^{n}$ for which (1) is verified almost everywhere in $[a, b]$. We now introduce our definitions and state the main results.

Definition 1 The system (1) is Globally Asymptotically Controllable (abbreviated GAC) if the two following conditions are satisfied.

Attractivity: For each $\xi \in \mathbb{R}^{n}$ there is a control $u:[0, \infty[\rightarrow U$ that drives $\xi$ asymptotically to 0 .

Lyapunov Stability: For each $\epsilon>0$, there is a $\delta>0$ such that for each $\xi \in \mathbb{R}^{n}$ with $\|\xi\| \leq \delta$ there is a control $u:[0, \infty[\rightarrow U$ that drives $\xi$ asymptotically to 0 and such that the corresponding trajectory $x(\cdot)$ satisfies $\| x(t \| \leq \epsilon$ for all $t \geq 0$. 
A basic tool for the study of the globally asymptotically controllable systems is the controlLyapunov function. In general a smooth Lyapunov function fails to exists, the obstruction being the Brockett condition [4]. This fact leads to the consideration of nonsmooth control-Lyapunov functions where the decrease condition is stated in terms of proximal subgradients.

Definition 2 A control-Lyapunov function (CLF) for the system (1) is a continuous, positive definite and proper function $V: \mathbb{R}^{n} \rightarrow \mathbb{R}$ such that there exists a continuous positive definite $W: \mathbb{R}^{n} \rightarrow \mathbb{R}$ with the property that:

$$
\forall x \in \mathbb{R}^{n} \backslash\{0\}, \forall \zeta \in \partial_{P} V(x), \min _{u \in U}\langle\zeta, f(x, u)\rangle \leq-W(x) .
$$

Here $\partial_{P} V(x)$ refers to the proximal subdifferential of $V$ at $x$ (which may be empty); see [8].

THEOREM 1 If the system (1) is GAC, then there exists a control-Lyapunov function semiconcave on $\mathbb{R}^{n} \backslash\{0\}$.

This fact extends the well-known result of Sontag [12] and brings an affirmative answer to a conjecture (existence of a locally Lipschitz CLF) that has been attributed to Sontag and Sussmann. Furthermore, the semiconcavity is new, and provides a useful tool for the construction of discontinuous feedbacks which will stabilize the system in the Euler sense. Assume given a feedback $u: \mathbb{R}^{n} \rightarrow U$, a partition $\pi=\left\{0=t_{0}<t_{1}<\cdots\right\}$ of $\left[0, \infty\left[\right.\right.$, and an $x_{0} \in \mathbb{R}^{n}$. For each $i, i=0,1,2, \cdots$, recursively solve

$$
\left.\dot{x}=f(x(t)), u\left(x_{i}\right)\right), \quad t \in\left[t_{i}, t_{i+1}\right]
$$

using as initial value $x\left(t_{i}\right)$, the endpoint of the solution of the preceding interval (and starting with $\left.x(0)=x_{0}\right)$. The $\pi$-trajectory of $\dot{x}=f(x, u(x))$ starting from $x_{0}$ is the function $x(\cdot)$ so obtained. An Euler solution of $\dot{x}=f(x, u(x)), x(0)=x_{0}$ is a uniform limit of $\pi$-trajectories of the same system with $\operatorname{diam}(\pi) \rightarrow 0$.

THEOREM 2 Assume that the system (1) is GAC, then there exists a feedback $u: \mathbb{R}^{n} \rightarrow U$ for which the system $\dot{x}=f(x, u(x))$ is globally asymptotically stabilisable in the Euler sense.

While this theorem is known [5] [6], we are able to give a simplified proof by invoking the semiconcavity in Theorem 1.

THEOREM 3 If moreover, the system is affine in the control, then the feedback can be chosen to be continuous in an open dense set. In this case, the system $\dot{x}=f(x, u(x))$ is globally asymptotically stabilisable in the Carathéodory sense.

\section{Introduction}

Donnons nous un système commandé de la forme générale

$$
\dot{x}(t)=f(x(t), u(t)),
$$

où l'état $x$ vit dans $\mathbb{R}^{n}$, la commande $u$ dans un espace métrique compact $U$ et où l'application $f$ de $\mathbb{R}^{n} \times U$ dans $\mathbb{R}^{n}$ est localement Lipschitzienne en $(x, u)$. Nous supposerons de plus que le point origine se distingue de tous les autres; il est point d'équilibre pour le système, c'est à dire $f(0,0)=0$ pour une certaine commande "0" appartenant à $U$. Une trajectoire de ce système est un arc absolument continu $x(\cdot):[a, b] \rightarrow \mathbb{R}^{n}$ associé à une commande $u(\cdot):[a, b] \rightarrow U$ pour 
laquelle $x(\cdot)$ est solution de (1) presque partout. L'objet de cet article est d'étudier les systèmes pour lesquels chaque état de $\mathbb{R}^{n}$ peut être conduit asymptotiquement à l'origine, et ce de manière uniforme. Donnons une définition plus précise de cette propriété.

DÉFINITION 1 Le système est dit Globalement Asymptotiquement Commandable à l'origine (noté $G A C)$ si les deux conditions suivantes sont réalisées.

Attractivité: Pour tout $\xi \in \mathbb{R}^{n}$, il existe une commande $u:[0, \infty[\rightarrow U$ qui mène asymptotiquement la trajectoire solution de (1) à l'origine.

Stabilité Lyapunov: Pour tout $\epsilon>0$, il existe $\delta>0$ tel que si $\xi \in \mathbb{R}^{n}$ est tel que $\|\xi\| \leq \delta$, alors il existe une commande $u:[0, \infty[\rightarrow U$ qui mène la trajectoire $x(\cdot)$ solution de (1) $\grave{a}$ l'origine et telle que $\forall t \geq 0, \| x(t \| \leq \epsilon$.

Il est naturel de se demander si on peut associer à un tel système un retour d'état $u(\cdot): \mathbb{R}^{n} \rightarrow U$ qui rendrait l'équation différentielle $\dot{x}=f(x, u(x))$ globalement asymptotiquement stable. Cette note présente quelques résultats concernant cette question; elle reste dans l'approche déjà utilisée dans [5] et $[6]$.

\section{Fonction Lyapunov de commande}

La construction d'un retour d'état stabilisant passe très souvent par l'utilisation d'une fonction Lyapunov de commande. Ce concept généralise celui introduit par Lyapunov dans le cas des équations différentielles ordinaires.

DÉFINITION 2 Une fonction Lyapunov de commande (FLC) associée au système (1) est une fonction $V: \mathbb{R}^{n} \rightarrow \mathbb{R}$, continue, définie positive (i.e. $V(0)=0$ et $V>0$ sur $\mathbb{R}^{n} \backslash\{0\}$ ) et propre pour laquelle il existe une fonction $W: \mathbb{R}^{n} \rightarrow \mathbb{R}$, continue définie positive qui vérifie:

$$
\forall x \in \mathbb{R}^{n} \backslash\{0\}, \forall \zeta \in \partial_{P} V(x), \min _{u \in U}\langle\zeta, f(x, u)\rangle \leq-W(x) .
$$

Le $\partial_{P} V(x)$ de la définition signifie sous-différentiel (ou sous-gradient) proximal de $V$ en $x$, i.e. l'ensemble des $\zeta \in \mathbb{R}^{n}$ tels qu'il existe $\delta, \sigma>0$ pour lesquels

$$
V(y)-V(x)+\sigma\|y-x\|^{2} \geq\langle\zeta, y-x\rangle \quad \forall y \in x+\delta B
$$

il s'agit d'un ensemble convexe, éventuellement vide. Il permet, lorsque $V$ est localement lipschitzienne, de définir le gradient généralisé de Clarke $\partial_{C} V(x)$ de la manière suivante:

$$
\partial_{C} V(x):=\operatorname{conv} \partial_{L} V(x) \text { avec } \partial_{L} V(x):=\left\{\lim \zeta_{i} ; \zeta_{i} \in \partial_{P} V\left(x_{i}\right), x_{i} \rightarrow x\right\} .
$$

Ces objets s'adaptent assez bien aux règles du calcul différentiel; nous renvoyons le lecteur à [8] pour de plus amples détails. Il faut signaler que $\partial_{P} V(x), \partial_{L} V(x)$ (sous-différentiel limite) et $\partial_{C} V(x)$ coïncident avec $\{\nabla V(x)\}$ dans le cas d'une fonction $V$ lisse (au moins $C^{2}$ ). Cependant, l'obtention d'une fonction Lyapunov lisse n'est pas envisageable dans le cas de systèmes commandés GAC quelconques. L'obstacle à cette régularité est constitué par la condition de Brockett [4], nécessaire à l'existence d'une FLC lisse ou de manière équivalente, à l'existence d'un retour d'état stabilisant assez régulier (i.e. continu hors de l'origine ou de type Filippov ou Krasovskii); on peut aller voir [7] et [3] pour une discussion sur ce sujet. De nombreux systèmes GAC, comme le nonholonomic integrator [5] par exemple, ne vérifient pas cette condition et ne possèdent par conséquent ni FLC lisse, ni retour d'état stabilisant suffisamment régulier. La définition ci-dessus permet de combler 
cette lacune et de définir un objet fournissant une généralisation naturelle de la fonction Lyapunov de commande au cas non lisse. En outre, le théorème suivant assure l'existence d'une fonction Lyapunov de commande dans le cas d'un système commandé GAC quelconque.

THÉORÈme 1 Supposons le système (1) GAC, alors il existe une fonction Lyapunov de commande semi-concave sur $\mathbb{R}^{n} \backslash\{0\}$.

Ce théorème apporte la réponse à une conjecture attribuée à Sontag et Sussmann; la preuve en est donnée dans [11]. Par ailleurs, la semi-concavité nous rapproche d'une régularité lisse; en effet, une fonction semiconcave peut être vue localement comme la somme d'une fonction lisse et d'une fonction concave. Ceci implique en particulier le lemme suivant (on note $\partial^{P} V(x):=-\partial_{P}(-V)(x)$ le surdifférentiel proximal de $V$ en $x$ ).

Lemme 1 Soit $V$ une application semi-concave sur un ouvert $\Omega \subset \mathbb{R}^{n}$. Alors pour tout $x \in \Omega$,

$$
\partial^{P} V(x)=\partial_{C} V(x) ;
$$

de plus, le $\sigma$ de l'inégalité (3) peut être pris uniforme sur les compacts de $\Omega$.

\section{Retours d'état stabilisants}

Dans la mesure où des retours d'états stabilisants suffisamment réguliers n'existent généralement pas, il nous faut donner un sens aux solutions de l'équation différentielle $\dot{x}=f(x, u(x))$ lorsque la fonction $x \longmapsto f(x, u(x))$ n'est pas continue; nous introduisons pour cela une variante de la notion de solution Euler, bien connue en jeux différentiel [10]. Notre approche diffère de celle de Sussmann [13] qui avait le premier remarqué que les retours d'état réguliers ne suffisent pas à stabiliser tous les systèmes GAC, et donc avait proposé une alternative discontinue dans le cas de systèmes analytiques. Signalons aussi qu'une autre approche (voir [9]) complètement différente existe; elle permet de construire des retours d'états stabilisant lisses, mais ils sont en contre-partie dépendants du temps.

Soit donc une boucle fermée $u: \mathbb{R}^{n} \longmapsto U$ non nécessairement continue et un état $x_{0} \in \mathbb{R}^{n}$. Considérons le problème de Cauchy

$$
\dot{x}(t)=f\left(x(t), u(x(t)), \quad x(0)=x_{0},\right.
$$

sur l'intervalle $\left[0, \infty\left[\right.\right.$. Donnons-nous une partition $\pi=\left\{a=t_{0}<t_{1}<t_{2}<\cdots\right\}$ de $[0, \infty[$; on note son diamètre $\operatorname{diam}(\pi):=\max \left|t_{i+1}-t_{i}\right|$. Nous considérons dans un premier temps le problème de Cauchy $\dot{x}=f\left(x, u\left(x_{0}\right)\right), x\left(t_{0}\right)=x_{0}$ sur l'intervalle $\left[t_{0}, t_{1}\right]$. Celui-ci possède une solution unique $x(t)$ sur $\left[t_{0}, t_{1}\right]$ (nous supposons ici que l'existence d'une solution sur tout l'intervalle est vérifiée, ce qui sera le cas par la suite); nous notons par ailleurs $x_{1}:=x\left(t_{1}\right)$. Puis on réitère cette opération en considérant le problème de Cauchy $\dot{x}=f\left(x, u\left(x_{1}\right)\right), x\left(t_{1}\right)=x_{1}$ sur $\left[t_{1}, t_{2}\right]$. On définit ainsi par itérations successives un arc appelé $\pi$-trajectoire de (4) sur $[0, \infty[$.

DÉFINITION 3 Nous appellerons solution Euler de (4) tout arc $x$ limite uniforme de $\pi_{i}$-trajectoires de (4) associées à des partitions $\pi_{i}$ pour lesquelles $\operatorname{diam}\left(\pi_{i}\right) \rightarrow_{i \rightarrow \infty} 0$.

Notons qu'une $\pi$-trajectoire peut, dans le cas où elle exploserait en temps fini, ne pas être définie sur $[0, \infty[$. Cependant, toutes les solutions Euler issus de nos retours d'état stabilisants seront définies globalement. Par ailleurs, nous dirons que le système $\dot{x}=f(x, u(x))$ est globalement 
asymptotiquement stabilisable (GAS) au sens des solutions Euler (resp. des solutions Carathéodory) si il vérifie les deux conditions d'attractivité et de stabilité Lyapunov adaptées aux solutions Euler (resp. aux solutions Carathéodory).

THÉORÈme 2 Supposons le système (1) GAC, alors, il existe un retour d'état $u: \mathbb{R}^{n} \rightarrow U$ qui rend le systeme $\dot{x}=f(x, u(x))$ GAS au sens des solutions Euler.

Ce théorème est démontré dans [5] et [6]. Ici, l'utilisation d'une fonction Lyapunov semi-concave simplifie considérablement la preuve.

Esquisse de la preuve.- D'après le théorème 1 et par définition du sous-différentiel limite, il existe une fonction Lyapunov de commande $V$ semi-concave sur $R^{n} \backslash\{0\}$ (elle est par définition continue sur $\mathbb{R}^{n}$ ) telle que:

$$
\forall x \in \mathbb{R}^{n} \backslash\{0\}, \forall \zeta \in \partial_{L} V(x), \min _{u \in U}\langle\zeta, f(x, u)\rangle \leq-W(x)<0 .
$$

Ainsi, pour tout $x \in \mathbb{R}^{n} \backslash\{0\}$, choisissons arbitrairement $\zeta \in \partial_{L} V(x)$ puis posons $u(x):=u$ où $u$ réalise le minimum dans l'inégalité ci-dessus (le choix de $u$ n'est pas forcément unique non plus). Nous définissons de cette manière une application $u: \mathbb{R}^{n} \rightarrow U$ (on pose bien-sûr $\left.u(0)=0\right)$ qui n'a aucune raison d'être continue, ni même mesurable.

Maintenant, soit $\pi$ une partition de $\left[0, \infty\left[\right.\right.$ et $x(\cdot)$ une $\pi$-trajectoire de $(4)$ sur $[0, b]$ (où $x_{0} \in \mathbb{R}^{n}$ ). Par le lemme 1, il existe $\sigma$ tel que pour tout $x$ dans un voisinage de la trajectoire $x(\cdot), \forall \zeta \in \partial_{L} V(x)$,

$$
V(y)-V(x) \leq\langle\zeta, f(x, u(x))\rangle+\sigma\|y-x\|^{2}, \quad \forall y \in \mathcal{V}(x)
$$

Ainsi, en appliquant cette inégalité aux $x_{i}$ de la $\pi$-trajectoire, on trouve

$$
V\left(x_{n}\right)-V\left(x_{0}\right) \leq-\sum_{i=0}^{n-1}\left(t_{i+1}-t_{i}\right) W\left(x_{i}\right)+O(\operatorname{diam}(\pi)) .
$$

Ainsi pour toute solution Euler $x(\cdot)$ sur $[0, b]$, on obtient par passage à la limite:

$$
V(x(t))-V\left(x_{0}\right) \leq-\int_{0}^{t} W(x(s)) d s, \quad \forall t \in[0, b] .
$$

Cette dernière inégalité démontre que les solutions Euler de (4) restent dans le compact $\{x: V(x) \leq$ $\left.V\left(x_{0}\right)\right\}$; elles seront donc par conséquent définies sur $[0, \infty[$. Les deux propriétés d'attractivité et de Lyapunov stabilité proviennent quant à elles du fait que pour toute solution Euler $x(\cdot)$ de $(4)$, on a $\int_{0}^{+\infty} W(x(s)) d s<\infty$.

Lorsque le système considéré est affine en la commande, c'est à dire lorsqu'il s'écrit $\dot{x}=f(x, u)=$ $f_{0}(x)+\sum_{i=1}^{p} u_{i} f_{i}(x)$ (où la commande $u=\left(u_{1}, \cdots, u_{p}\right)$ évolue dans un compact de $\mathbb{R}^{p}$ ), nous avons la possibilité de construire un retour d'état beaucoup plus régulier; il est stabilisant pour les solutions Carathéodory. Nous retrouvons en quelque sorte un résultat très récent de Ancona et Bressan [2] qui démontrent qu'en fait tout système GAC possède un retour d'état (lisse par morceaux) stabilisant au sens des solutions Carathéodory. Leur démonstration reste néanmoins totalement différente, elle ne repose pas sur l'existence d'une fonction Lyapunov non-lisse.

THÉORÈME 3 Si le systême est affine en la commande et GAC, alors le retour d'état stabilisant peut être choisi continu sur un ouvert dense et dans ce cas, le système $\dot{x}=f(x, u(x))$ est GAS au sens des solutions Carathéodory. 
Idée de la preuve.- Comme précédemment, le théorème 1 donne l'existence d'une fonction Lyapunov de commande $V$ semi-concave sur $R^{n} \backslash\{0\}$ telle que:

$$
\forall x \in \mathbb{R}^{n} \backslash\{0\}, \forall \zeta \in \partial_{L} V(x), \min _{u \in U}\langle\zeta, f(x, u)\rangle \leq-W(x)<0 .
$$

Nous pouvons alors considérer l'ensemble suivant:

$$
\mathcal{D}:=\left\{x \in \mathbb{R}^{n} \backslash\{0\} \text { tel que } \exists u \in U, \forall \zeta \in \partial_{C} V(x),\langle\zeta, f(x, u)\rangle<-\frac{W(x)}{2}\right\} .
$$

La fonction multivoque $x \mapsto \partial_{C} V(x)$ étant semi-continue supérieurement, il est clair que $\mathcal{D}$ est un ensemble ouvert. Par ailleurs, par un théorème de densité [8], le sous-différentiel proximal de $V$ est non vide sur un ensemble dense. Par conséquent, comme $V$ est semi-concave, $\partial_{P} V(x)$ et $\{\nabla V(x)\}$ coïncident sur ce même ensemble dense; cette propriété fournit la densité de $\mathcal{D}$. Maintenant, par le théorème de sélection de Michael, comme le système est affine en la commande, on peut construire une sélection continue $u: \mathcal{D} \rightarrow U$ telle que pour tout $x \in \mathcal{D}, \forall \zeta \in \partial_{C} V(x),\langle\zeta, f(x, u(x))\rangle \leq$ $-W(x) / 2$. On peut alors étendre par densité, $u$ à tout $\mathbb{R}^{n}$ de manière à ce que $(x, u(x))$ reste dans $\operatorname{adh}(\operatorname{Graph}(u))$ et $u(0)=0$. Cette construction reste dans le cadre de celle proposée dans le théorème précédent, ainsi le système (4) est GAS pour les solutions Euler. Bien que $x \mapsto f(x, u(x))$ ne soit pas continue, des solutions Carathéodory vont exister; les solutions Euler en font partie. En fait, par un résultat sur l'ensemble des singularités des fonctions semi-concaves [1], on montre que les solutions Euler sont aussi des solutions au sens de Carathéodory, c'est à dire qu'elles vérifient $\dot{x}(t)=f(x(t), u(x(t))$ presque partout. Pour finir, par une utilisation classique d'un théorème des accroissements finis non lisse, on démontre qu'en fait, le système (4) est GAS au sens des solutions Carathéodory.

REMARQUE 1 Il faut par ailleurs noter que les retours d'états construits dans les deux théorèmes sont robustes pour certaines perturbations correspondant à des partitions presque uniforme [5].

Remerciements. Je tiens à remercier Francis Clarke pour son aide précieuse.

\section{Références}

[1] G. Alberti, L. Ambrosio, and P. Cannarsa. On the singularities of convex functions. Manuscripta Math., 76(3-4):421-435, 1992.

[2] F. Ancona and A. Bressan. Patchy vector fields and asymptotic stabilization. ESAIM Control Optim. Calc. Var., 4:445-471 (electronic), 1999.

[3] Z. Artstein. Stabilization with relaxed controls. Nonlinear Analysis TMA, 7:1163-1173, 1983.

[4] R.W. Brockett. Asymptotic stability and feedback stabilization. In R.W. Brockett, R.S. Millman, and H.J. Sussmann, editors, Differential Geometric Control Theory, pages 181-191. Birkhäuser, Boston, 1983.

[5] F.H. Clarke, Yu.S. Ledyaev, L. Rifford, and R.J. Stern. Feedback stabilization and Lyapunov functions. A paraitre.

[6] F.H. Clarke, Yu.S. Ledyaev, E.D. Sontag, and A.I. Subbotin. Asymptotic controllability implies feedback stabilization. I.E.E.E. Trans. Aut. Control, 42:1394-1407, 1997.

[7] F.H. Clarke, Yu.S. Ledyaev, and R.J. Stern. Asymptotic stability and smooth Lyapunov functions. J. Differential Equations, 149:69-114, 1998. 
[8] F.H. Clarke, Yu.S. Ledyaev, R.J. Stern, and P.R. Wolenski. Nonsmooth Analysis and Control Theory. Graduate Texts in Mathematics, vol. 178. Springer-Verlag, New York, 1998.

[9] J-M. Coron. Global asymptotic stabilization for controllable systems without drift. Math. Cont. Sig. Sys., 5:295-312, 1992.

[10] N.N. Krasovskii and A.I. Subbotin. Game-Theoretical Control Problems. Springer-Verlag, 1988.

[11] L. Rifford. Existence of lipschitz and semiconcave control-Lyapunov functions. Soumis.

[12] E.D. Sontag. A Lyapunov-like characterization of asymptotic controllability. SIAM J. Control and Optim., 21:462-471, 1983.

[13] H. J. Sussmann. Subanalytic sets and feedback control. J. Differential Equations, 31(1):31-52, 1979 . 